\title{
Complicações em transplantes endoteliais realizados por estagiários de córnea
}

\section{Complications in endothelial keratoplasty performed by cornea fellows}

Nicolas Cesário Pereira ${ }^{1}$, Mônica Araújo ${ }^{1}$, Natércia Trindade Pinto ${ }^{1}$, Pedro Bertino Moreira ${ }^{1}$, Guilherme Andrade do Nascimento Rocha ${ }^{1}$, Gustavo Souza Moura ${ }^{1}$, Luciene Barbosa de Sousa ${ }^{1}$

\section{RESUMO}

Objetivo: Reportar complicações precoces numa série de transplantes endoteliais realizados por estagiários de córnea.

Métodos: Revisão retrospectiva dos transplantes endoteliais realizados por estagiários decórnea do segundo ano. Descritas as complicações precoces e respectivo tratamento. Resultados: Foram realizados 34 transplantes endoteliais pelos estagiários de córnea entre julho de 2007 e agosto de 2008. Destes, 29 foram realizados pela técnica de "Descemet stripping endothelial keratoplasty" e 5 "Descemet stripping automated endothelial keratoplasty". Foram realizadas 15 cirurgias combinadas, 14 associadas a facoemulsificação com implante de lente intraocular e 1 associada a facectomia com implante de lente intraocular. A principal indicação foi por distrofia de Fuchs com 18 casos, 11 foram indicados por ceratopatia bolhosa após cirurgia de catarata, 4 por falência primária após transplante endotelial e 1 por distrofia endotelial congênita hereditária. A complicação mais encontrada foi descolamentos do botão doado em 8 olhos (23,5\%). Falência primária foi encontrado em 7 olhos (20,6\%). Glaucoma agudo por bloqueio pupilar foi encontrado em 1 paciente $(2,9 \%)$.

Conclusões: Oalto número de complicações encontradas nestas cirurgias, queforam as primeiras de um grupo de 6 estagiários de córnea, mostra a longa curva de aprendizado para este procedimento cirúrgico. A orientação das primeiras cirurgias por cirurgiões mais experientes pode diminuir a curva de aprendizado e a taxa de complicações.

Descritores: Transplante de córnea; Lâmina limitante posterior; Epitélio posterior/ cirurgia; Ceratoplastia endotelial com remoção da lâmina limitante posterior; Complicações pós-operatórias; Procedimentos cirúrgicos oftalmológicos/efeitos adversos

\begin{abstract}
Purpose: To report early complications in endothelial keratoplasty performed by cornea fellows.

Methods: Retrospective study of endothelial keratoplasty performed by second-year cornea fellows. Described the early complications and its treatments.

Results: Thirty four endothelial keratoplasty were performed by cornea fellows, from July 2007 to August 2008. From this, 29 were Descemet stripping endothelial keratoplasty and 5 Descemet stripping automated endothelial keratoplasty; 14 combined with phacoemulsification and 1 with extracapsular cataract surgery. The main indication was Fuchs' dystrophy (18 cases), followed by bullous keratopathy (11 cases), primary failure after endothelial keratoplasty (4 cases) and congenital hereditary endothelial dystrophy (1 case). Main surgery complication was donor button detachment in 8 eyes (23.5\%), followed by primary graft failure in 7 eyes (20.6\%) and acute glaucoma after pupillary block in 1 eye (2.9\%).

Conclusions: The complication rate in this series, which were the first ever in a group of six cornea fellows, was high. This shows the steep learning curve for this surgical procedure. The assistance of an experienced surgeon could help to achieve a less steep learning curve with a lower complication rate.
\end{abstract}

Keywords: Corneal transplantation; Descemet membrane; Endothelium, corneal/ surgery; Descemet stripping endothelial keratoplasty; Postoperative complications; Ophthalmologic surgical procedures/adverse effects

\section{INTRODUÇÃO}

As disfunções do endotélio corneano tiveram o transplante penetrante como tratamento de escolha no século $X X^{(1-5)}$. Na última década o transplante endotelial se tornou uma boa alternativa, por oferecer uma reabilitação visual melhor e mais rápida, com mínima alteração do poder refrativo da córnea transplantada, mínimo astigmatismo induzido, eliminação das complicações induzidas pela sutura e deiscência tardia da incisão, além de menor necessidade de cuidados pós-operatórios ${ }^{(6-10)}$

O transplante endotelial é uma técnica de transplante de córnea que substitui apenas as camadas posteriores da córnea ${ }^{(6-10)}$. Com isso é possível realizar uma substituição seletiva da córnea com doenças restritas ao endotélio. É realizada através de um sistema fechado, que diminui e pode em alguns casos eliminar a necessidade de incisões ou suturas na superfície corneana ${ }^{(6-10)}$.
O primeiro transplante endotelial realizado com sucesso foi descrito por Charles Tillett em 1956, com o botão posterior suturado no leito receptor, mas não foi uma técnica difundida ${ }^{(11)}$. Em 1998 Gerrit Melles et al. descreveu o "posterior lamellar keratoplasty" (PLK), que foi a primeira técnica de transplante endotelial realizada com o botão doado sem suturas, com suporte de uma bolha de $\operatorname{ar}^{(6,12)}$. Mark Terry et al. fez algumas modificações no PLK e em 2001 difundiu a técnica cirúrgica como "deep lamellar endothelial keratoplasty" (DLEK), que consiste na retirada de lamela estromal posterior do receptor através de túnel escleral, com a inserção do botão doado na bolsa criada anteriormente ${ }^{(10)}$. A necessidade de realizar a dissecção manual do leito receptor e da córnea doada dificulta a técnica cirúrgica, que foi pouco difundida.

Após a descrição original da retirada seletiva da membrana de Descemet receptora por Melles et al. ${ }^{(13,14)}$, Price et al. introduziu e
Submetido para publicação: 17 de dezembro de 2012

Aceito para publicação: 26 de maio de 2013

Trabalho realizado no Hospital Oftalmológico de Sorocaba - Sorocaba (SP), Brasil.

${ }^{1}$ Médico, Hospital Oftalmológico de Sorocaba - Sorocaba (SP), Brasil.
Financiamento: Não houve financiamento para este trabalho.

Divulgação de potenciais Conflitos de Interesse: N.C.Pereira, Nenhum; M.Araújo, Nenhum; N.T.Pinto, Nenhum; P.B.Moreira, Nenhum; G.A.N.Rocha, Nenhum; G.S.Moura, Nenhum; L.B.de Sousa, Nenhum.

Endereço para correspondência: Nicolas Cesário Pereira, Rua Nabeck Shiroma, 210, Sorocaba (SP) - 18031-060 - E-mail: nicolascepe@gmail.com

Estudo aprovado pelo Comitê de Ética em Pesquisa do Hospital Oftalmológico de Sorocaba (SP) sob parecer número 023/2010. 
popularizou o "Descemet stripping endothelial keratoplasty" (DSEK)(15,16). A retirada seletiva da membrana de Descemet com endotélio receptor, sem estroma associado, facilitou o preparo da córnea receptora e tornou a técnica mais reprodutível. O preparo da córnea doada ainda é realizado manualmente no DSEK ${ }^{(15,16)}$. Em 2006 foi reportado a utilização de microcerátomo para preparar a córnea doada e foi denominado "Descemet stripping automated endothelial keratoplasty" (DSAEK) ${ }^{(17)}$. A técnica cirúrgica se tornou mais fácil e reprodutível, sendo amplamente difundida. Atualmente é a técnica de transplante endotelial com o maior número de publicações e é a mais realizada pelos cirurgiões de córnea em todo o mundo.

Apesar de ser uma técnica cirúrgica reprodutível e com ótimos resultados, o DSEK/DSAEK não fornece o máximo do potencial visual do paciente ${ }^{(15)}$. Em busca de uma qualidade visual superior e uma restauração da anatomia corneana mais natural, em 2006 Gerrit Mellles descreveu o "Descemet membrane endothelial keratoplasty" $(\mathrm{DMEK})^{(18)}$. Nessa técnica cirúrgica, a córnea receptora é preparada com o "stripping" da mesma forma que o DSEK/DSAEK, mas a córnea doada consiste apenas da membrana de Descemet e endotélio, sem estroma associado. Os resultados visuais são superiores e com uma reabilitação visual mais rápida ${ }^{(19-21)}$. Mas é uma técnica cirúrgica mais difícil, com número maior de complicações nas primeiras publicações ${ }^{(19-21)}$. Poucos cirurgiões no mundo realizam essa técnica com bons resultados e baixo índice de complicações.

Os estudos publicados nas diferentes técnicas de transplante endotelial têm sugerido vantagens importantes em relação às complicações da ceratoplastia penetrante ${ }^{(6-10)}$. Essas vantagens incluem realizar a cirurgia em sistema fechado, diminuindo o risco de hemorragia expulsiva e aumentando a segurança dos procedimentos combinados ${ }^{(6-8,10)}$. A superfície corneana se mantém praticamente inalterada, reduzindo astigmatismo irregular e melhorando a previsibilidade do poder refrativo da córnea com melhor acurácia da biometria nos casos de implante de lente intraocular associados ${ }^{(9,10)}$. Não existem as complicações relacionadas a sutura como vascularização, infecção e deiscência pós trauma ${ }^{(1-5)}$. Não existe comprometimento da inervação com menos complicações relacionadas à superfície corneana ${ }^{(8,10)}$. Além de existir boa densidade e função endotelial, existe menor risco de rejeição ${ }^{(7,8,15,22-24)}$. Todas estas vantagens têm contribuído para uma reabilitação visual mais rápida e segura ${ }^{(7,9,10)}$.

Apesar de todas as vantagens do transplante endotelial, existem complicações relacionadas a este procedimento e uma curva de aprendizado. Revisamos as complicações precoces e intraoperatórias que ocorreram nos primeiros transplantes endoteliais realizados pelos estagiários de córnea do segundo ano do Hospital Oftalmológico de Sorocaba.

\section{MÉTODOS}

Foram analisados de forma retrospectiva os prontuários de 29 pacientes que foram submetidos a transplante endotelial no Hospital Oftalmológico de Sorocaba, no período entre julho de 2007 e agosto de 2008. Destes pacientes, 1 teve transplante endotelial bilateral e em 4 foi realizado retransplante endotelial após falência primária, totalizando 34 transplantes endoteliais. Os resultados foram analisados com análise estatística descritiva. O estudo foi aprovado pelo Comitê de Ética em Pesquisa da instituição sob parecer número 023/2010.

Todos os transplantes foram realizados por seis estagiários de córnea do segundo ano, cada um com uma experiência média de cem transplantes penetrantes. Os transplantes endoteliais deste estudo foram os primeiros realizados por estes estagiários. Estes foram os primeiros estagiários de córnea treinados em transplante endotelial no Hospital Oftalmológico de Sorocaba.

Todas as cirurgias foram realizadas com bloqueio peribulbar. As incisões foram corneanas (nas cirurgias combinadas com facoemulsificação) ou túneis esclerais e tiveram pequenas variações no tamanho, medindo entre 4 e $5 \mathrm{~mm}$. Em todas as córneas receptoras foi retirado seletivamente apenas membrana de Descemet e endotélio, com a técnica de "stripping", com metilcelulose para manter a câmara anterior. "Scraping" ou raspagem da periferia do leito estromal receptor foi realizada em todos os casos, por ter sido descrito melhorar a aderência do botão(25).

Existiram variações das técnicas cirúrgicas empregadas na preparação e inserção do botão doado. Foi utilizada dissecção manual do botão doado (DSEK) em 29 cirurgias e dissecção automatizada com microcerátomo (DSAEK) em cinco cirurgias. A inserção do botão doado foi realizada com pinça em 21 cirurgias e com a técnica da sutura do botão em 13 cirurgias.

No final da cirurgia, com o botão posicionado, uma bolha de ar grande foi mantida na câmara anterior por 10 a 15 minutos. Enquanto isso a pupila era dilatada e depois a bolha de ar diminuída para 5 a $8 \mathrm{~mm}$, para evitar bloqueio pupilar no pós-operatório. Os pacientes foram recomendados a olhar para cima em decúbito dorsal por no mínimo 1 hora após a cirurgia.

No pós-operatório foi utilizado quinolona de quarta geração quatro vezes ao dia por 7 dias e acetato de prednisolona a 1\% oito vezes ao dia nos primeiros quinze dias, diminuindo sua frequência de acordo com a resposta clínica.

\section{RESULTADOS}

Foram realizados 34 transplantes endoteliais pelos estagiários de córnea entre julho de 2007 e agosto de 2008. Destes, 29 foram realizados pela técnica de DSEK e 5 DSAEK. Um dos pacientes que foi tentado realizar preparação do botão doado com microcerátomo (DSAEK) teve um corte irregular, sendo necessária dissecção manual (DSEK) que ocorreu sem intercorrências.

Foram realizadas 15 cirurgias combinadas (44,1\%), 14 com facoemulsificação e 1 com facectomia extracapsular. A principal indicação foi por distrofia de Fuchs com 18 casos, 11 foram indicados por ceratopatia bolhosa após cirurgia de catarata, 4 por falência primária após transplante endotelial e 1 por distrofia endotelial congênita hereditária.

Descolamentos do botão doado foi a complicação mais encontrada. Dos 34 transplantes endoteliais realizados, 8 olhos (23,5\%) apresentaram algum tipo de descolamento do botão. Encontramos descolamento total no primeiro dia pós-operatório em 3 casos (8,8\%) (Figura 1). Todos os casos foram reposicionados no mesmo dia com injeção de $C_{3} F_{8}$ a $10 \%$ na câmara anterior e evoluíram bem (Figuras 2 e 3). A acuidade visual dos pacientes que tiveram complicações e evoluíram bem encontra-se na tabela 1. Cinco olhos (14,7\%) apresentaram no sétimo pós-operatório pequenos descolamentos do botão.

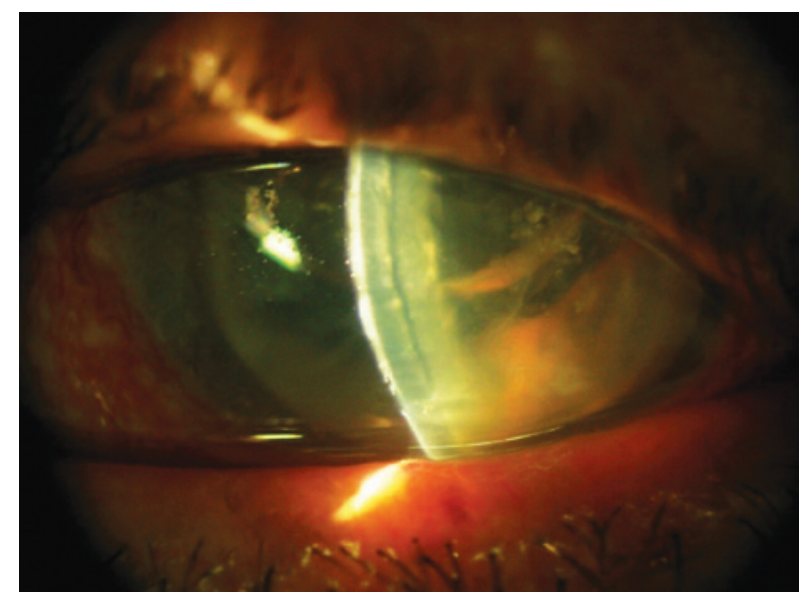

Figura 1. Paciente com descolamento no primeiro dia após DSAEK. 


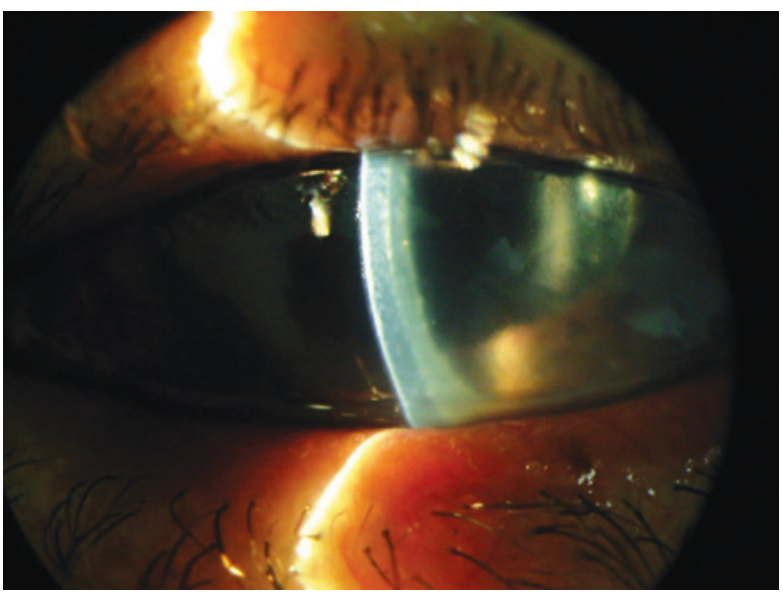

Figura 2. Paciente da figura 1 com botão colado, 7 dias após injeção de $C_{3} F_{8}$ a $10 \%$.

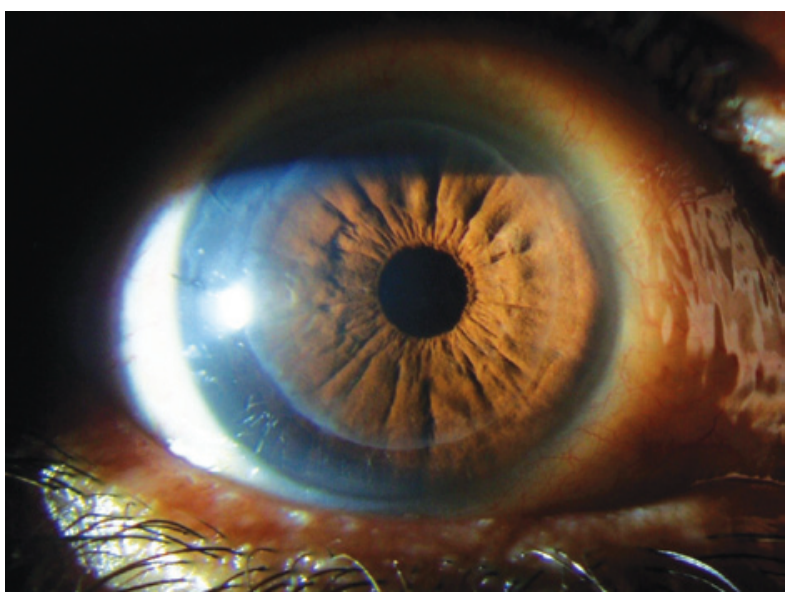

Figura 3. Paciente com córnea transparente após DSEK+faco, que teve descolamento do botão e foi reposicionado com injeção de $\mathrm{C}_{3} \mathrm{~F}_{8}$ a $10 \%$.

Destes, 1 foi tratado com injeção de ar no sétimo dia pós-operatório e evoluiu bem. Os outros 4 tiveram tratamento expectante e destes, 3 apresentaram piora do edema e do descolamento e evoluíram para falência primária. Apenas 1 evoluiu bem, mas o pequeno descolamento inferior levou a um edema epitelial localizado sintomático, que melhorou após realização de micropuntura localizada. Este paciente estava com o botão doado totalmente colado no quinto mês de pós-operatório. Dos 3 que evoluíram com falência primária, 2 foram submetidos a retransplante endotelial e 1 a transplante penetrante. Dos 8 olhos que apresentaram descolamento do botão, 6 foram em cirurgias combinadas com facoemulsificação.

Quatro olhos evoluíram com falência primária sem descolamento do botão. Destes, 3 foram em cirurgias combinadas com facoemulsificação. Dois foram submetidos a retransplante endotelial e 2 a transplante penetrante. Os quatro evoluíram bem. No total foram 7 falências primárias $(20,6 \%), 4$ foram submetidos a retransplante endotelial e 3 a transplante penetrante. Um paciente (2,9\%) apresentou glaucoma agudo por bloqueio pupilar no segundo pós-operatório, foi realizada intervenção no mesmo dia com iridectomia e reposicionamento da bolha de ar. Um olho teve hérnia de íris pela incisão no quarto pós-operatório que foi reduzida cirurgicamente no mesmo dia.

O número de complicações em transplantes endoteliais simples e em cirurgias combinadas encontra-se na tabela 2.
Tabela 1. Acuidade visual dos pacientes com complicações que tiveram boa evolução

\begin{tabular}{|c|c|c|c|c|}
\hline Paciente & Complicação & Acuidade visual & $\begin{array}{l}\text { Tipo de } \\
\text { cirurgia }\end{array}$ & $\begin{array}{c}\text { Acuidade } \\
\text { visual } \\
\text { pré- } \\
\text { operatória }\end{array}$ \\
\hline 1 & Descolado 1으 & 20/40 c/c 4º mês PO & DSEK + faco & $20 / 200 \mathrm{c} / \mathrm{c}$ \\
\hline 2 & Descolado 1으 PO & CD 3 m 50 $\mathrm{PO}^{*}$ & DSEK + faco & $\mathrm{CD} 2 \mathrm{~m} \mathrm{c} / \mathrm{c}$ \\
\hline 3 & Descolado 1ํ PO & CD $1 \mathrm{~m} \mathrm{20 \circ} \mathrm{PO}^{*}$ & DSAEK & $\mathrm{CD} 1 \mathrm{~m} \mathrm{c} / \mathrm{c}$ \\
\hline 4 & Descolado 7으 PO & 20/40 c/c 3 mês PO & DSEK + faco & $20 / 200 \mathrm{c} / \mathrm{c}$ \\
\hline 5 & Descolado 7으 PO & 20/40 s/c 3 mês PO & DSEK + faco & $20 / 160 \mathrm{c} / \mathrm{c}$ \\
\hline 6 & Glaucoma agudo & 20/50 s/c 5 mês PO & DSEK & $20 / 100 \mathrm{c} / \mathrm{c}$ \\
\hline 7 & Hérnia de íris & 20/200 s/c 3ำ mês PO & DSEK + faco & $20 / 400 \mathrm{c} / \mathrm{c}$ \\
\hline
\end{tabular}

* Os pacientes 2 e 3 perderam seguimento com 50 e 20 dias de pós-operatório, respectivamente.

$\mathrm{PO}=$ pós-operatório; $\mathrm{c} / \mathrm{c}=$ com correção; $\mathrm{s} / \mathrm{c}=$ sem correção; DSEK = "Descemet stripping endothelial keratoplasty"; DSAEK= "Descemet stripping automated endothelial keratoplasty"; faco= facoemulsificação com implante de lente intraocular.

Tabela 2. Complicações em transplantes endoteliais simples e em cirurgias combinadas

\begin{tabular}{|c|c|c|c|}
\hline Cirurgia & $\begin{array}{c}\text { Com complicação } \\
\text { n (\%) }\end{array}$ & $\begin{array}{c}\text { Sem complicação } \\
\text { n (\%) }\end{array}$ & $\begin{array}{l}\text { Total } \\
\text { n (\%) }\end{array}$ \\
\hline $\begin{array}{l}\text { Simples } \\
14 \text { DSEK e } \\
5 \text { DSAEK }\end{array}$ & $5(26,31)$ & $14(73,69)$ & $19(100)$ \\
\hline $\begin{array}{l}\text { Combinada } \\
14 \text { DSEK + faco e } \\
1 \text { DSEK + fec }\end{array}$ & $9(60)$ & $6(40)$ & $15(100)$ \\
\hline
\end{tabular}

DSEK = "Descemet stripping endothelial keratoplasty"; DSAEK = "Descemet stripping automated endothelial keratoplasty"; faco= facoemulsificação com implante de lente intraocular: $f e c=$ facectomia extracapsular com implante de lente intraocular.

\section{DISCUSSÃO}

As vantagens do transplante endotelial foram amplamente difundidas e relatadas nos últimos anos ${ }^{(16,25-28)}$. Apesar de todas as vantagens, existem complicações relacionadas a este procedimento e uma curva de aprendizado. As principais complicações incluem descolamentos e falências do enxerto ${ }^{(16,25-28)}$. Suas prováveis causas incluem presença de viscoelástico, fluido ou ar na interface, trauma excessivo do botão doado e problemas com o preparo e inserção do botão doado ${ }^{(16,25-28)}$.

Neste estudo as principais complicações descritas foram descolamentos e falências, como descrito em outras publicações ${ }^{(16,25-28)}$. Encontramos 8 descolamentos (23,5\%), sendo que apenas $3(8,8 \%)$ eram descolamentos totais e foram observados no primeiro dia de pós-operatório. Estes 3 olhos foram operados por estagiários que estavam realizando pela primeira vez este tipo de cirurgia. Outros estudos também demonstraram inexperiência com o procedimento resultando em descolamentos do botão(28). Gorovoy et al. demonstrou uma taxa de $25 \%$ em 16 olhos $^{(29)}$. Mearza et al. teve descolamentos em $82 \%$ de 11 olhos $^{(30)}$. Price et al. demonstrou uma taxa de descolamentos de $50 \%$ nos 10 primeiros casos, que reduziu para $13 \%$ nos próximos 126 casos e foi para 6\% nos últimos $64 \operatorname{casos}^{(16)}$. Terry et al. teve taxa de $50 \%$ de descolamento nos primeiros 4 casos, que foi reduzido para $4 \%$ nos próximos 100 olhos ${ }^{(27)}$. Estes estudos relatam maior taxa de descolamentos no início da curva de aprendizado destes cirurgiões. Com maior experiência do cirurgião e com a evolução da técnica cirúrgica, foi possível uma taxa de descolamentos menor. Em uma revisão realizada pela Academia Americana de Oftalmologia, que incluiu as principais publicações em DSEK/DSAEK, relatou uma 
média de $14,5 \%$ de descolamentos ${ }^{(31)}$. A taxa de $23,5 \%$ de descolamentos encontrada foi maior que a média das publicações. $O$ início da curva de aprendizado pode ter sido um dos fatores nesta taxa aumentada. Em todas as cirurgias foi utilizado metilcelulose, que é um viscoelástico dispersivo difícil de ser totalmente aspirado antes da inserção do botão. Viscoelástico na interface pode ter sido outro fator importante na maior taxa de descolamentos nesse estudo. Estudos com baixa taxa de descolamentos utilizaram viscoelástico coesivo para realizar a cirurgia ${ }^{(15,16,25,27,32)}$.

Tivemos 7 (20,6\%) falências primárias. Destas, 2 aconteceram com estagiários que estavam realizando este tipo de cirurgia pela primeira vez. Mearza et al. teve $45 \%$ de falência em 11 olhos ${ }^{(30)}$. Koenig et al. demonstrou $10 \%$ de falência em 21 olhos ${ }^{(33)}$. Price et al. teve $4 \%$ de falência em $200 \operatorname{casos}^{(16)}$. Suh et al. teve taxas muito parecidas com o nosso estudo, com $17 \%$ de falências e $23 \%$ de descolamentos ${ }^{(28)}$. A média das taxas de falências entre as publicações encontrada na revisão realizada pela Academia Americana de Oftalmologia foi de $5 \%{ }^{(31)}$. Encontramos uma taxa maior, com provável causa o trauma excessivo do endotélio doado no início da curva de aprendizado.

Ocorreu um caso de glaucoma agudo por bloqueio pupilar (2,9\%), que aconteceu no segundo transplante endotelial de um dos estagiários. O bloqueio pupilar ocorreu por manutenção de uma bolha muito grande na câmara anterior no fim da cirurgia. Terry et al. recomenda uma bolha pequena e móvel para prevenir bloqueio pupilar e não teve este tipo de complicação nas séries publicadas ${ }^{(25,27,32)}$.

As cirurgias combinadas foram responsáveis por $75 \%$ dos descolamentos e $85,7 \%$ das falências encontradas neste estudo. A taxa de complicações que encontramos em transplantes endoteliais simples foi de $26,3 \%$ enquanto que a taxa encontrada em cirurgias combinadas foi de $60 \%$. Este estudo teve grande número de cirurgias combinadas (44,1\%), enquanto outros estudos foram realizados com poucas ou nenhuma cirurgia combinada ${ }^{(15-17,27,28)}$. Este número maior de procedimentos combinados pode ter contribuído com a taxa de complicações nesse estudo.

Por ser um procedimento relativamente novo com técnicas diferentes e ainda em evolução, encontramos taxas de complicações muito variáveis entre os estudos. Nossos resultados mostram uma alta taxa de complicações nas primeiras cirurgias dos estagiários, o que demonstra uma longa curva de aprendizado para esta técnica.

Em um estudo que compara transplante endotelial realizado por cirurgião experiente e cirurgiões em treinamento, foi encontrado mesma taxa de complicações ${ }^{(32)}$. Todas as cirurgias foram acompanhadas pelo mesmo cirurgião experiente (Mark Terry), com orientação verbal durante todo o procedimento. Quando necessário o cirurgião experiente assumiu a cirurgia para minimizar os riscos. Esse estudo concluiu que apesar da longa curva de aprendizado do transplante endotelial, é possível diminuir as complicações com uma técnica cirúrgica adequada associada a orientação intraoperatória por cirurgião experiente.

\section{CONCLUSÕES}

"Descemet stripping endothelial keratoplasty" e "Descemet stripping automated endothelial keratoplasty" são opções de tratamento cirúrgico para as doenças endoteliais. Apresentam algumas vantagens em relação ao transplante penetrante, mas possuem uma curva de aprendizado longa com potenciais complicações. Cirurgiões iniciantes nesta técnica devem passar por treinamento em laboratório e devem ter suas primeiras cirurgias orientadas por cirurgiões experientes. Assim se espera diminuir a curva de aprendizado e suas possíveis complicações.

\section{REFERÊNCIAS}

1. Abou-Jaoude ES, Brooks M, Katz DG, Van Meter WS. Spontaneous wound dehiscence after removal of single continuous penetrating keratoplasty suture. Ophthalmology. 2002;109(7):1291-6; discussion 1297.
2. Tseng SH, Lin SC, Chen FK. Traumatic wound dehiscence after penetrating keratoplasty: clinical features and outcome in 21 cases. Cornea. 1999:18(5):553-8.

3. Stechschulte SU, Azar DT. Complications after penetrating keratoplasty. Int Ophthalmol Clin. 2000;40(1):27-43. Review.

4. Akova YA, Onat M, Koc F, Nurozler A, Duman S. Microbial keratitis following penetrating keratoplasty. Ophthalmic Surg Lasers. 1999;30(6):449-55.

5. Confino J, Brown SI. Bacterial endophthalmitis associated with exposed monofilament sutures following corneal transplantation. Am J Ophthalmol. 1985;99(2):111-3.

6. Melles GR, Eggink FA, Lander F, Pels E, Rietveld FJ, Beekhuis WH, et al. A surgical technique for posterior lamellar keratoplasty. Cornea. 1998;17(6):618-26.

7. Terry MA. Deep lamellar endothelial keratoplasty (DLEK): pursuing the ideal goals of endothelial replacement. Eye (Lond). 2003;17(8):982-8.

8. Terry MA. A new approach for endothelial transplantation: deep lamellar endothelial keratoplasty. Int Ophthalmol Clin. 2003;43(3):183-93.

9. Terry MA, Ousley PJ. Rapid visual rehabilitation after endothelial transplants with deep lamellar endothelial keratoplasty (DLEK). Cornea. 2004;23(2):143-53.

10. Terry MA, Ousley PJ. Endothelial replacement without surface corneal incisions or sutures: topography of the deep lamellar endothelial keratoplasty procedure. Cornea. 2001;20(1):14-8

11. Tillett CW. Posterior lamellar keratoplasty. Am J Ophthalmol. 1956:41(3):530-3.

12. Melles GR, Lander F, Beekhuis WH, Remeijer L, Binder PS. Posterior lamellar keratoplasty for a case of pseudophakic bullous keratopathy. Am J Ophthalmol. 1999; 127(3):340-1.

13. Melles GR, Lander F, Rietveld FJ. Transplantation of Descemet's membrane carrying viable endothelium through a small scleral incision. Cornea. 2002:21(4):415-8.

14. Melles GR, Wijdh RH, Nieuwendaal CP. A technique to excise the descemet membrane from a recipient cornea (descemetorhexis). Cornea. 2004:23(3):286-8.

15. Price MO, Price FW Jr. Descemet's stripping with endothelial keratoplasty: comparative outcomes with microkeratome-dissected and manually dissected donor tissue. Ophthalmology. 2006;113(11):1936-42.

16. Price FW Jr, Price MO. Descemet's stripping with endothelial keratoplasty in 200 eyes: early challenges and techniques to enhance donor adherence. J Cataract Refract Surg. 2006:32(3):411-8

17. Gorovoy MS. Descemet-stripping automated endothelial keratoplasty. Cornea. 2006;25(8):886-9. Comment in Cornea. 2006;25(8):879-81.

18. Melles GR, Ong TS, Ververs B, van der Wees J. Descemet membrane endothelia keratoplasty (DMEK). Cornea. 2006; 25(8): 987-90

19. Melles GR, Ong TS, Ververs B, van der Wees J. Preliminary clinical results of Descemet membrane endothelial keratoplasty. Am J Ophthalmol. 2008;145(2):222-7.

20. Ham L, Dapena I, van Luijk C, van der Wees J, Melles GR. Descemet membrane endothelial keratoplasty (DMEK) for Fuchs endothelial dystrophy: review of the first 50 consecutive cases. Eye (Lond). 2009;23(10):1990-8.

21. Price MO, Giebel AW, Fairchild KM, Price FW Jr. Descemet's membrane endothelia keratoplasty: prospective multicenter study of visual and refractive outcomes and endothelial survival. Ophthalmology. 2009;116(12):2361-8.

22. Allan BD, Terry MA, Price FW Jr. Price MO, Griffin NB, Claesson M. Corneal transplant rejection rate and severity after endothelial keratoplasty. Cornea. 2007;26(9):1039-42.

23. Price MO, Jordan CS, Moore G, Price FW Jr. Graft rejection episodes after Descemet stripping with endothelial keratoplasty: part two: the statistical analysis of probability and risk factors. Br J Ophthalmol. 2009;93(3):391-5.

24. Anshu A, Price MO, Price FW Jr. Risk of corneal transplant rejection significantly reduced with Descemet's membrane endothelial keratoplasty. Ophthalmology. 2012; 119(3):536-40.

25. Terry MA, Shamie N, Chen ES, Hoar KL, Friend DJ. Endothelial keratoplasty a simplified technique to minimize graft dislocation, iatrogenic graft failure, and pupillary block. Ophthalmology. 2008;115(7):1179-86.

26. Terry MA, Ousley PJ. Deep lamellar endothelial keratoplasty: early complications and their management. Cornea. 2006;25(1):37-43.

27. Terry MA, Hoar KL, Wall J, Ousley P. Histology of dislocations in endothelial keratoplasty (DSEK and DLEK): a laboratory-based, surgical solution to dislocation in 100 consecutive DSEK cases. Cornea. 2006:25(8):926-32

28. Suh LH, Yoo SH, Deobhakta A, Donaldson KE, Alfonso EC, Culbertson WW, et al. Complications of Descemet's stripping with automated endothelial keratoplasty: survey of 118 eyes at One Institute. Ophthalmology. 2008;115(9):1517-24.

29. Gorovoy MS. Descemet-stripping automated endothelial keratoplasty. Cornea. 2006 25(8):886-9.

30. Mearza AA, Qureshi MA, Rostron CK. Experience and 12-month results of Descemetstripping endothelial keratoplasty (DSEK) with a small-incision technique. Cornea. 2007;26(3):279-83. Comment in Cornea. 2007;26(10):1292-3; author reply 1293.

31. Lee WB, Jacobs DS, Musch DC, Kaufman SC, Reinhart WJ, Shtein RM. Descemet's stripping endothelial keratoplasty: safety and outcomes: a report by the American Academy of Ophthalmology. Ophthalmology. 2009:116(9):1818-30.

32. Chen ES, Terry MA, Shamie N, Hoar KL, Phillips PM, Friend DJ. Endothelial keratoplasty: vision, endothelial survival, and complications in a comparative case series of fellows vs attending surgeons. Am J Ophthalmol. 2009;148(1):26-31.

33. Koenig SB, Covert DJ. Early results of small-incision Descemet's stripping and automated endothelial keratoplasty. Ophthalmology. 2007;114(2):221-6. 